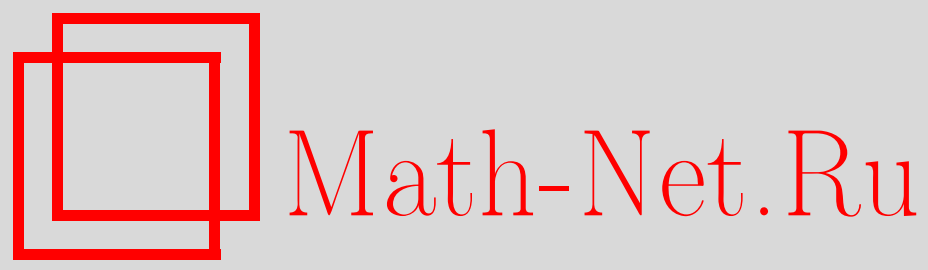

В. Ю. Овсиенко, К. Роже, Расширения группы Вирасоро и алгебры Вирасоро с помощью модулей

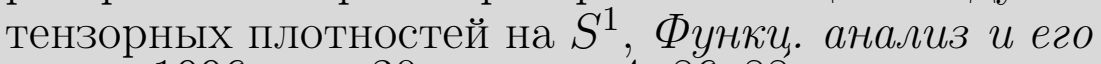
прил., 1996, том 30, выпуск 4, 86-88

DOI: https://doi.org/10.4213/faa559

Использование Общероссийского математического портала MathNet.Ru подразумевает, что вы прочитали и согласны с пользовательским соглашением http://www. mathnet.ru/rus/agreement

Параметры загрузки:

IP : 52.87 .193 .239

26 апреля 2023 г., 15:17:33

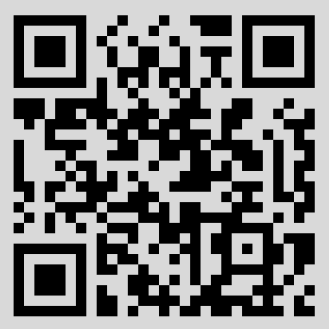


плотны в ней, то имеет место равенство

$$
\mathbf{K}\left(H_{A}\right)=\overline{\bigcup_{\beta \in \mathbf{B}} K^{\beta}\left(H_{A}\right)},
$$

аде черта означает замыкание в исходной норме.

Автор благодарен А. С. Мищенко, Е. В. Троицкому и М. Франку за полезные обсуждения.

\title{
ЛиТЕРАТУРА
}

1. Paschke W. L. Can. J. Math., 26, 1272-1280 (1974). 2. Мищенко A. С., Фоменко A. Т. Изв. АН СССР. Сер. матем., 43, 831-859 (1979). 3. Kasparov G. G. J. Operator Theory, 4, 133-150 (1980). 4. Frank M. Geometrical Aspects of Hilbert $C^{*}$-Modules. Preprint No. 22, Københavns Universitet, 1993. 5. Troitsky E. V. Some aspects of geometry of operators in Hilbert modules. Preprint No. 173, Ruhr-Universität Bochum, 1994.

Московский государственный университет им. М. В. Ломоносова

\section{Расширения группы Вирасоро и алгебры Вирасоро с помощью модулей тензорных плотностей на $S^{1}$}

\author{
(c) 1996. В. Ю. Овсиенко, К. Роже
}

1. Группа $\operatorname{Diff}^{+}\left(S^{1}\right)$ (диффеоморфизмов окружности, сохраняющих ориентацию) обладает единственным (с точностью до изоморфизма) нетривиальным центральным расширением, задаваемым с помошью коцикла Серстона-Ботта (см. [1]) $B(\Phi, \Psi)=\int_{S^{1}} \log \left((\Phi \circ \Psi)^{\prime}\right) d \log \left(\Psi^{\prime}\right)$, где $\Phi, \Psi \in \operatorname{Diff}^{+}\left(S^{1}\right), \Psi^{\prime}=$ $d \Psi / d x, x$ - параметр на $S^{1}$. Полученная группа называется группой БоттаВирасоро.

Соответствующая алгебра Ли называется алгеброй Вирасоро. Она задается единственным (с точностью до изоморфизма) нетривиальным центральным расширением алгебры Ли $\operatorname{Vect}\left(S^{1}\right)$ (гладких векторных полей на окружности) с помощью коцикла Гельфанда-Фукса (см. [3]) $\omega(f, g)=\int_{S^{1}} f^{\prime} g^{\prime \prime} d x$, где $f=$ $f(x) d / d x, g=g(x) d / d x$.

2. Обозначим через $\mathscr{F}_{\lambda} \operatorname{Diff}^{+}\left(S^{1}\right)$ - и $\operatorname{Vect}\left(S^{1}\right)$-модуль тензорных плотностей $a=a(x)(d x)^{\lambda}, a(x) \in C^{\infty}\left(S^{1}\right)$, степени $\lambda$ на $S^{1}$.

В этой работе мы рассматриваем расширения группы $\operatorname{Diff}^{+}\left(S^{1}\right)$ и алгебры Ли $\operatorname{Vect}\left(S^{1}\right)$ с помощью модулей тензорных плотностей на $S^{1}$. Найденные группы и алгебры Ли являются аналогами группы и алгебры Вирасоро.

Мы вычисляем группу $H_{c}^{2}\left(\operatorname{Diff}^{+}\left(S^{1}\right) ; \mathscr{F}_{\lambda}\right)$ дифференщируемых в смысле ВанЭста когомологий, что означает классификацию расширений, заданных дифференциальными операторами. В результате получены четыре новые бесконечно- 
мерные группы Ли. Мы предъявим точные формулы для нетривиальных коциклов на $\operatorname{Diff}^{+}\left(S^{1}\right)$.

Teopema 1. $H_{c}^{2}\left(\operatorname{Diff}^{+}\left(S^{1}\right) ; \mathscr{F}_{\lambda}\right)= \begin{cases}\mathbb{R}, & \lambda=0,1,2,5,7, \\ 0, & \lambda \neq 0,1,2,5,7 .\end{cases}$

3. Опишем явно 2-коциклы на $\operatorname{Diff}^{+}\left(S^{1}\right)$, задающие нетривиальные классы когомологий. Напомним, что отображения

$$
l(\Phi)=\log \left(\Phi^{\prime}\right), \quad d l(\Phi)=\frac{\Phi^{\prime \prime}}{\Phi^{\prime}} d x, \quad S(\Phi)=\left[\frac{\Phi^{\prime \prime \prime}}{\Phi^{\prime}}-\frac{3}{2}\left(\frac{\Phi^{\prime \prime}}{\Phi^{\prime}}\right)^{2}\right](d x)^{2}
$$

определяют нетривиальные 1 -коциклы на $\operatorname{Diff}^{+}\left(S^{1}\right)$ со значениями в $\mathscr{F}_{0}, \mathscr{F}_{1}$, $\mathscr{F}_{2}$ соответственно. Коцикл $S$ - это так называемая производная Шварца, а $d l$ - логарифмическая производная.

Определим оператор $D: \mathscr{F}_{\lambda} \rightarrow \mathscr{F}_{\lambda+1}$ формулой $D\left(a(x)(d x)^{\lambda}\right)=a^{\prime}(x)(d x)^{\lambda+1}$.

ТеОрема 2. Нетривиальнье 2-кочикльь

$$
\begin{gathered}
B_{1}(\Phi, \Psi)=(l(\Phi) \circ \Psi) \cdot d l(\Psi), \quad B_{2}(\Phi, \Psi)=(l(\Phi) \circ \Psi) \cdot S(\Psi), \\
B_{5}(\Phi, \Psi)=(S(\Phi) \circ \Psi) \cdot D S(\Psi)-S(\Psi) \cdot D(S(\Phi) \circ \Psi), \\
B_{7}(\Phi, \Psi)=2\left|\begin{array}{cc}
S(\Phi) \circ \Psi & S(\Psi) \\
D^{3}(S(\Phi) \circ \Psi) & D^{3}(S(\Psi))
\end{array}\right|-9\left|\begin{array}{cc}
D(S(\Phi) \circ \Psi) & D(S(\Psi)) \\
D^{2}(S(\Phi) \circ \Psi) & D^{2}(S(\Psi))
\end{array}\right| \\
-\frac{32}{3}(S(\Psi)+S(\Phi \circ \Psi)) B_{5}(\Phi, \Psi),
\end{gathered}
$$

а также $B_{0}(\Phi, \Psi)=B(\Phi, \Psi)$ (коцикл Серстона-Ботта) задают базис группьи когомологии $H_{c}^{2}\left(\operatorname{Diff}^{+}\left(S^{1}\right) ; \mathscr{F}_{\lambda}\right)$, где $\lambda=0,1,2,5,7$.

Первый из этих коциклов - коцикл Серстона-Ботта - означает здесь постоянную функцию на $S^{1}$.

4. Классификация нетривиальных расширений алгебры Ли $\operatorname{Vect}\left(S^{1}\right)$ дается следуюшим утверждением Цудзисита [5] (см. [2, с. 147]):

$$
H^{2}\left(\operatorname{Vect}\left(S^{1}\right) ; \mathscr{F}_{\lambda}\right)= \begin{cases}\mathbb{R}^{2}, & \lambda=0,1,2, \\ \mathbb{R}, & \lambda=5,7, \\ 0, & \lambda \neq 0,1,2,5,7 .\end{cases}
$$

Опишем 2-коциклы на $\operatorname{Vect}\left(S^{1}\right)$ со значениями в $\mathscr{F} \lambda$, представляющие нетривиальные классы когомологий.

Теорема 3. Следующие восемь нетривиальных 2-кочиклов: кочикл Гельфанда-Фукса $c_{0}(f, g)=\omega(f, g)$, а также

$$
\begin{gathered}
\bar{c}_{0}(f, g)=f g^{\prime}-f^{\prime} g, \quad c_{1}(f, g)=\left(f^{\prime} g^{\prime \prime}-f^{\prime \prime} g^{\prime}\right) d x, \\
\bar{c}_{1}(f, g)=\left(f g^{\prime \prime}-f^{\prime \prime} g\right) d x, \quad c_{2}(f, g)=\left(f^{\prime} g^{\prime \prime \prime}-f^{\prime \prime \prime} g^{\prime}\right)(d x)^{2}, \\
\bar{c}_{2}(f, g)=\left(f g^{\prime \prime \prime}-f^{\prime \prime \prime} g\right)(d x)^{2}, \quad c_{5}(f, g)=\left(f^{\prime \prime \prime} g^{(I V)}-f^{(I V)} g^{\prime \prime \prime}\right)(d x)^{5}, \\
c_{7}(f, g)=\left(2\left(f^{\prime \prime \prime} g^{(V I)}-f^{(V I)} g^{\prime \prime \prime}\right)-9\left(f^{(I V)} g^{(V)}-f^{(V)} g^{(I V)}\right)\right)(d x)^{7}
\end{gathered}
$$

образуют базис группы когомологий $H^{2}\left(\operatorname{Vect}\left(S^{1}\right) ; \mathscr{F}_{\lambda}\right)$, где $\lambda=0,1,2,5,7$. 
ЗАмЕчАНИЯ. 1. Коциклы $c_{0}, c_{1}, c_{2}, c_{5}, c_{7}$ на алгебре Ли $\operatorname{Vect}\left(S^{1}\right)$ отвечают групповым коциклам $B_{0}, B_{1}, B_{2}, B_{5}, B_{7}$. Коциклы $\bar{c}_{0}, \bar{c}_{1}, \bar{c}_{2}$ не могут быть проинтегрированы до коциклов на группе $\operatorname{Diff}^{+}\left(S^{1}\right)$.

2. Коцикл $c_{5}$ был найден в [4].

Обозначим через $\mathfrak{g}_{i}$ алгебру Ли, заданную коциклом $c_{i}$, а через $\overline{\mathfrak{g}}_{i}$ алгебру Ли, заданную коциклом $\bar{c}_{i}$.

5. Опишем центральные расширения алгебр Ли $\overline{\mathfrak{g}}_{i}$ и $\mathfrak{g}_{i}$. Каждая из алгебр Ли $\mathfrak{g}_{1}, \mathfrak{g}_{2}, \mathfrak{g}_{5}, \mathfrak{g}_{7}$ и $\overline{\mathfrak{g}}_{0}, \overline{\mathfrak{g}}_{1}, \overline{\mathfrak{g}}_{2}$ имеет нетривиальное центральное расширение, заданное коциклом $c((f, a),(g, b))=\omega(f, g)$.

ПРЕДЛОЖЕНИЕ 1. Существуют еще ровно два нетривиальных чентральных расширения:

1) алгебры Ли $\overline{\mathfrak{g}}_{1}$, заданное коциклом $c((f, a),(g, b))=\int_{S^{1}}(f b-g a) d x$;

2) алгебры Ли $\mathfrak{g}_{1}$, заданное кочиклом $c((f, a),(g, b))=\int_{S^{1}}\left(f^{\prime} b-g^{\prime} a\right) d x$.

Рассмотрим теперь центральные расширения полупрямого произведения.

Предложение 2. $H^{2}\left(\operatorname{Vect}\left(S^{1}\right) \triangleright \mathscr{F}_{\lambda} ; \mathbb{R}\right)= \begin{cases}\mathbb{R}^{3}, & \lambda=0,1, \\ \mathbb{R}, & \lambda \neq 0,1 .\end{cases}$

В случаях $\lambda=0$ и $\lambda=1$ сушествуют два нетривиальных коцикла, неэквивалентных продолжению коцикла Гельфанда-Фукса. Их можно задать в виде $c((f, a),(g, b))=\int_{S^{1}}\left(f^{\prime \prime} b-g^{\prime \prime} a\right) d x$ и $c((f, a),(g, b))=\int_{S^{1}}(a d b-b d a)$.

\section{ЛитератУРА}

1. Bott R. Enseign. Math., 23, No. 3-4, 209-220 (1977). 2. Фукс Д. Б. Когомологии бесконечномерных алгебр Ли. Наука, М., 1984. 3. Гельфанд И. М., Фукс Д. Б. Функц. анализ и его прил., 2, вып. 4, 92-93 (1968). 4. Овсиенко В. Ю., Роже К. УМH, 47, вып. 6, 141-194 (1992). 5. Tsujishita T. Proc. Japan Acad. A, 53, No. 4, $134-138$ (1977).

C. N. R. S., Centre de Physique Théorique, Marseille

Université Claude Bernard-Lion I

Поступило в редакцию 20 августа 1995 г. В переработанном виде 24 января 1996 г.

УдК 517.9

\section{О спектральных свойствах задачи Орра-Зоммерфельда при исчезающей вязкости}

(C) 1996. С. А. Степин

При исследовании спектральных свойств сингулярно возмущенных операторов приходится отказываться от методов аналитической теории возмущений, в которой основную роль играет непрерывность резольвенты по параметру в смысле сходимости по норме. Примером такого рода служит задача на собственные значения для оператора вида $P+\varepsilon Q$, где $P$ и $Q-$ дифференциальные операторы

^ Работа выполнена при поддержке Международного научного фонда (грант NCI300). 